\title{
Foreword
}

\section{Nernst, polarography, and what next?}

\author{
Hubert H. Girault
}

The origin of electroanalysis is usually dated back to the end of the $19^{\text {th }}$ century, and among all the scientists active in the field at that time most students nowadays mainly remember the name of Walther Hermann Nernst (1864-1941). Perhaps, when considering the fact that most electroanalytical methods involve electrolyte solutions, students may also be familiar with the name of Svante Arrhenius (1859-1927).

In any case, attributing the advancement of science to only a few scientists is not recognising that progress in science is a collective effort based on communication, some creativity and many hours spent in the laboratory...

If we try to analyse what we have collectively done over 150 years in electroanalytical chemistry, we can distinguish four main achievements:

- Comprehension of conductivity phenomena in electrolyte solutions, at least in the linear regime where the current is proportional to the electric field.

- Comprehension of the potential distribution at polarised interfaces, where most students know the Gouy-Chapman model.

- Development of ion selective electrode technology despite a certain lack of theoretical understanding. The classical example is the $\mathrm{pH}$ electrode 1 that is widely

\footnotetext{
1 The chair of electrochemistry that I have the pleasure to occupy was created at the beginning of the twentieth century with the mission to study $\mathrm{pH}$
}

used in many fields of chemistry, but the working mecanisms remains somewhat obscure.

- Development of amperometric methods, including impedance spectroscopy and biosensors.

It is not the purpose of this preface to review all these fields as it has been done many times before. What I would prefer to discuss is more to analyse how we got where we are now, and to envisage what lies ahead in front of us.

In the first half of the twentieth century, the main emphasis was given to the study of electrolyte solutions. Thermodynamic analysis gave us the concept of ion activity; statistical mechanics gave us an idea on ion-ion interaction (Debye-Hückel theory) and ion pairing. From an experimental point of view, the main instrumentation used was the voltmeter and the Wheatstone bridge. This part of physical chemistry is not always taught anymore and perhaps some phenomena, such as the Wien effect that has disappeared from many textbooks, will be left to science historians.

Research in the second half of the twentieth century has been mainly technology driven. It should be recognised that progress in electrochemistry would not have been possible without the development of what was called electrotechnics, for instance the invention of the transistor, first

electrodes in relation to the acidity of wine produced in the Canton de Vaud. 
the amplifying vacuum tube ${ }^{2}$ then the solid state version. With electrotechnics came the potentiostat that has opened the road to polarography and more generally to amperometry. From this period, we have kept cyclic voltammetry that could be realised with an analog ramp generator and measured with a paper graph recorder or even with an oscilloscope. The oscilloscope at that time was a major tool for any electrochemists, as we always had an eye on the voltage or current signal not always trusting our instrumentation. If cyclic voltammetry has survived until now, it is perhaps due to the "beauty" of the cyclic voltammogram, a shape seen nowhere else in science, unlike the ubiquitous Gaussian peak response provided by so many techniques. For different reasons that include experimental difficulties and the toxicity of mercury, polarography has not survived to the $21^{\text {st }}$ century.

The second technological revolution for electrochemistry has been the development of digital instrumentation and computers in the eighties. The early days of this period seem so archaic nowadays but so close in a way $^{3}$. With digital instruments, we were able to apply any potential waveform and calculate plenty of Laplace transforms to analyse the current response. From simple potential steps to all sort of pulse methods (square wave, differential pulse, etc.), it was possible to design any input function to the electrochemical system, the transfer function of which providing the

\footnotetext{
2 I started my PhD in 1979 with a potentiostat from Chemical Electronics (UK) that was a big cube of $60 \mathrm{~cm} \times 60 \mathrm{~cm} \times 60 \mathrm{~cm}$ with of course analog meters. To unblock the needle of the meters after say an overload, a good kick in the side of this box was the only way to reset the instrument.

${ }^{3}$ Close enough for me to remember it well...
}

corresponding output.

The third technological revolution came with the scanning probes techniques such as AFM, STM, Kelvin probe, SECM, etc. For the first time, we have been able to look at an electrode with an atomic resolution.

From all this technological progress, what did we learn?

The answer could be: A lot but not that much...

Mass transfer controlled amperometry is a very mature field and even complex electrochemical reactions such as EC mechanisms are well characterised. Amperometric techniques are now routine, but their use at present has been rather limited, for example as a detection method in chromatography.

In potentiometry, the ISE response has not been fully characterised and improvement in theory and technology are still in progress.

On the charge transfer theory front, the progress since the early work of Gerischer, Dogonadze, Marcus et. al. has been incremental. Not to mention the present theories of ion transfer reactions across a liquid/liquid interface which are still very primitive.

I do not know if we have learnt a lot from scanning probes, but at least we got nice pictures...

Anyway, lack of understanding of phenomena at the fundamental level has never hindered applications. Electroanalytical applications have flourished over the recent decades. One of the most successful applications of amperometry has been the coupling with enzyme catalysis for the development of biosensors, in particular disposable electrodes. Billions of glucose strips are being produced every year.

Applications linked to the coupling with 
spectroscopy

such

as

electrochemi-luminescence, have also reached the multi million dollars mark.

When I teach electroanalytical chemistry ${ }^{4}$, I always include a chapter on electrophoresis. This electrochemical technique has been the workhorse of biologists for more than 50 years, and we should never forget that the human genome was sequenced by an electroanalytical method. Another technique that is widely used in electroanalytical chemistry is electrospray ionisation for mass spectrometry.

More general applications of electrochemistry can, of course, be found in the fields of energy storage and conversion, or in engineering science for example in micro (or nano) machining or in material corrosion.

Thus, if the $20^{\text {th }}$ century was technology driven, what will be driving research in electroanalytical chemistry over the next decades. Of course, as the French President Jacques Chirac once said :" The most difficult thing to predict is the future!".

My own feeling is that science will be more aimed at understanding nature and at solving problems of our modern society. Somehow our politicians want us to be useful. The general turn of chemistry and physics towards biology is not just a fashion, but it is due to the fact that all the tools developed during the technology driven period can now be used to tackle the very large complexity of biological systems. As far as electroanalytical chemistry is concerned, we have a gold mine to exploit for many years to come. Bioanalytical chemistry offers many challenges and

\footnotetext{
${ }^{4}$ See for example, Analytical and Physical Electrochemistry, H. Girault, EPFL Press/Marcel Dekker, 2004.
}

electrochemical methods will be useful both for separation and analysis. Because electroanalytical methods scale down very easily, coupling with micro-fluidics offers many possibilities, for example electrochemical immunoassays in microchips have a bright future. Also for understanding bioenergetics, the coupling between ion transfer processes and charge transfer reactions needs to be better understood. Of course, the Holy Grail for electrochemists is brain chemistry. Our brain is just a complex electrochemical computer, and electroanalytical methodology has a lot to offer to understand how information is generated, transmitted and stored. We can already see how micro-electrodes are increasingly used in brain research.

The second large domain of research will be linked to the global energy requirement of our society. Yet again, electrochemists have a lot to offer from bio fuel cells to molecular solar energy conversion.

For all these reasons, I believe that teaching electrochemical science and methodology is very important for all science students. In the same way that spectroscopy has been compulsory over the last few decades, I believe it would be beneficial for the advancement of science if science students had a good grasp of concepts such as potential distribution, diffusion potentials, space charged region, charge transfer reactions, $\mathrm{pH}$, ionic activity, ion pairs, etc. Unfortunately, these are often difficult concepts and one just needs to speak to our colleagues (say the organic chemists, not to mention the biologists) to see how many scientists are not educated in these fundamental concepts.

（ユベール・ジロー教授、ローザンヌ連邦 工科大学、スイス) 